\title{
Pacientes com astigmatismo submetidos à cirurgia de catarata: LIO tórica x LIO asférica?
}

\author{
Patients with astigmatism who underwent cataract surgery by phacoemulsification: \\ toric IOL $\mathrm{x}$ asferic IOL?
}

Emilio de Almeida Torres Netto ${ }^{1}$, Marina Carvalho Gulin ${ }^{1}$, Marcio Zapparoli $^{1}$, Hamilton Moreira ${ }^{2}$

\section{RESUMO}

Objetivos: Comparar a acuidade visual dos pacientes que foram submetidos à facoemulsificação com implante de LIO AcrySof ${ }^{\circledR}$ tórica versus LIO AcrySof ${ }^{\circledR}$ IQ e avaliar a redução da dioptria cilíndrica (DC) pós-operatória.

Métodos: Estudo analítico retrospectivo de 149 olhos submetidos à cirurgia de catarata por facoemulsificação, com astigmatismo ceratométrico regular, simétrico, com 1 dioptria ou mais. Foram divididos em dois grupos. O grupo tórica com 85 olhos e o grupo não tórica com 64 olhos. No pré-operatório foram avaliados dados topográficos do olho a ser operado e refração. No período pós-operatório foram revisados dados da refração e acuidades visuais com e sem correção.

Resultados: $\mathrm{O}$ astigmatismo corneano pré-operatório variou de 1,00 a 5,6 DC em ambos os grupos, sendo que no grupo tórica houve redução média de 1,37 DC $(p<0,001)$, quando comparado ao refracional. O grupo não tórica apresentou redução média de $0,16 \mathrm{DC}(p=0,057)$. Com relação a acuidade visual sem correção (AVSC), o grupo tórica apresentou 44 olhos $(51,7 \%)$ com AVSC de 0 logMAR (20/20) ou 0,1 logMAR (20/25), e o grupo não tórica apenas 7 olhos (10,93\%) com estas mesmas AVSC.

Discussão: Ficou bem evidenciado que os pacientes com astigmatismo ceratométrico significativo apresentam benefício visual com o implante de LIO tórica. A diminuição do uso de auxílios ópticos para distância pode ser possível a partir do momento que conseguimos corrigir com melhor precisão as aberrações do olho humano. Na atualidade, a facoemulsificação passou a ser além de uma cirurgia para restabelecimento funcional, um procedimento refracional.

Descritores: Astigmatismo/cirurgia; Implante de lente intraocular/métodos; Facoemulsificação; Extração de catarata; Acuidade visual

\begin{abstract}
Purposes: Compare the visual acuity of patients who underwent cataract surgery by phacoemulsification with $1 O L$ AcrySof $^{\circledR}$ toric implantation versus AcrySof ${ }^{\circledR} I Q$ and evaluate the reduction of cylindrical diopters (CD) in the postoperative period.

Methods: Analytical and retrospective study of 149 eyes with 1 or more diopters of regular symmetrical keratometric astigmatism, which underwent cataract surgery by phacoemulsification. The eyes were divided into two groups: the toric group with 85 eyes and the non-toric group with 64 eyes. In the pre-operative phase, topographic data and refraction of each eye to be operated were assessed. In the postoperative phase, refraction and visual acuity with and without correction were measured.

Results: The preoperative topographic astigmatism ranged from 1.00 to $5.6 \mathrm{DC}$ in both groups. Average reduction of $1.37 C D(p<0.001)$ and $0.16 C D(p=0.057)$ was obtained for the toric and non-toric group when compared to the refractive astigmatism, respectively. Considering visual acuity without correction (NCVA), the toric group presented 44 eyes (51.7\%) with NCVA of O logMAR (20/20) or 0.1 logMAR (20/25) and the toric group presented 7 eyes (10.93\%) with these same NCVA values. Discussion: The results show that patients with a significant keratometric astigmatism presented visual benefits with the toric IOL implantation. The reduction of the use of optical aids may be obtained provided aberrations of the human eye are corrected more accurately. Currently, phacoemulsification surgery has been used not only for functional improvement, but also as a refraction procedure.
\end{abstract}

Keywords: Astigmatism/surgery; Intraocularlens implantation/methods; Phacoemulsification; Cataract extraction; Visual acuity

\section{INTRODUÇÃO}

O termo astigmatismo vem de "a" que significa "falta de" e"stigma" que significa "um ponto". O astigmatismo ocorre quando os meridianos principais do olho têm poderes refrativos diferentes. Assim, no astigmatismo a imagem não atinge a retina em um único ponto focal, mas sim, sempre, em duas linhas focais. A medida do intervalo focal entre eles corresponde ao grau do astigmatismo ${ }^{(1,2)}$.

O astigmatismo regular tem os dois meridianos principais a ângulos retos um do outro, ou seja, a $90^{\circ}$. No astigmatismo irregular, os dois meridianos principais não se encontram perpendicularmente e, geralmente, é secundário (doença corneana, trauma, pterígio, subluxação do cristalino, cirurgia de catarata) $)^{(3,4)}$.
Sabe-se que o astigmatismo é mais comumente produzido pela toricidade da superfície anterior da córnea, embora sua etiologia, na maior parte das vezes, seja desconhecida. Se o meridiano de menor curvatura estiver em um ângulo entre $160^{\circ}$ e $20^{\circ} \mathrm{com}$ o plano horizontal, chamamos de astigmatismo a favor da regra; entre $70^{\circ}$ e $110^{\circ}$, astigmatismo contra a regra; e entre $21^{\circ}$ e $69^{\circ}$ e entre $111^{\circ}$ e $159^{\circ}$, astigmatismo oblíquo ${ }^{(3)}$.

No passado havia certa satisfação por parte do médico e paciente de realizar cirurgias sem intercorrências. Porém, o avanço tecnológico e as exigências impostas pelos pacientes, cada vez mais informados, conduziu-nos a aprimorar nossos resultados e fornecer além de boa acuidade visual, a correção refracional, com uma possível indepen-
Submetido para publicação: 18 de julho de 2012

Aceito para publicação: 27 de maio de 2013

Trabalho realizado no Hospital de Olhos do Paraná - Curitiba (PR), Brasil.

Médico, Hospital de Olhos do Paraná - Curitiba (PR) - Brasil.

Médico, Universidade Federal do Paraná; Faculdade Evangélica do Paraná; Hospital de Olhos do

Paraná - Curitiba (PR) - Brasil.
Financiamento: Não houve financiamento para este trabalho.

Divulgação de potenciais conflitos de interesse: E.A.T.Netto, Nenhum; M.C.Gulin, Nenhum; M.Zapparoli, Nenhum; H.Moreira, Nenhum.

Endereço para correspondência: Hamilton Moreira. Rua Coronel Dulcidio, 199 - 4 andar - Curitiba (PR) - 80420-170 - Brasil - E-mail: hamiltonmoreira@mac.com

Comitê de Ética em Pesquisa da Sociedade Evangélica Beneficente de Curitiba, protocolado sob número 4977/10. 
dência de óculos ${ }^{(5-12)}$. Neste sentido, a lente intraocular tórica vem a contribuir muito no manejo de pacientes com astigmatismo.

O objetivo deste estudo é comparar a acuidade visual e a redução do componente cilíndrico nos pacientes que foram submetidos à cirurgia de extração do cristalino por facoemulsificação com implante de lente intraocular (LIO) AcrySof ${ }^{\circledR}$ tórica versus LIO AcrySof ${ }^{\circledR} \mathrm{IQ}$.

\section{MÉTODOS}

Estudo analítico e retrospectivo baseado em análise de prontuários de pacientes submetidos à cirurgia de catarata por facoemulsificação no Hospital de Olhos do Paraná, no período de fevereiro de 2010 a setembro de 2011. Foi revisado um total de 1.453 cirurgias, sendo que 1.256 olhos foram submetidos a implante de lente LIO AcrySof ${ }^{\circledR}$ IQ $\left(\right.$ Alcon $\left.^{\circledR}\right)$, e 196 submetidos a implante de LIO AcrySof ${ }^{\circledR}$ tórica $\left(A \mid c o n{ }^{\circledR}\right)$. Os pacientes foram incluídos em dois grupos: Grupo tórica (olhos com implante de LIO AcrySof ${ }^{\circledR}$ tórica) e Grupo não tórica (olhos com implante de LIO AcrySof ${ }^{\circledR} \mathrm{IQ}$ ).

O comprimento axial foi determinado com ultrassonografia de imersão AScan (Ocuscan XP, Alcon ${ }^{\circledR}$ ). O cálculo do poder esférico da LIO foi determinado considerando o comprimento axial dos olhos estudados, sendo usada a formula Holladay para comprimentos axiais variando entre 22 a $24 \mathrm{~mm}$, a regra SRK-T foi utilizada nos olhos com mais de $24 \mathrm{~mm}$ e Hoffer Q usada nos olhos que apresentaram comprimento menor que $22 \mathrm{~mm}$, objetivando a emetropia. O cálculo do poder cilíndrico da lente e seu posicionamento foram determinados pelo seu fabricante levando em consideração a topografia computadorizada, poder esférico da LIO, posição da incisão e astigmatismo induzido pelo cirurgião. Este cálculo foi realizado pelo fabricante da LIO (Alcon ${ }^{\circledR}$ ) através do site www.acrysoftoriccalculator.com.

O critério de inclusão foi olhos que apresentavam, além de catarata, astigmatismo corneano maior ou igual a 1,00 D comprovado ao exame de topografia corneana computadorizada (Eye Sys Vista ${ }^{\circledR}$. Texas, USA) e que foram submetidos ao implante de alguma das LIO's citadas (AcrySof ${ }^{\circledR}$ tórica ou AcrySof ${ }^{\circledR} 1 Q$ ).

Foram excluídos todos os olhos que apresentaram qualquer comorbidade que comprometesse a acuidade visual final (retinopatia diabética, glaucoma com perda de campo visual além dos 30 graus centrais, sequelas de uveítes e conjuntivites, degeneração macular, ceratocone e astigmatismo irregular) e aqueles que não apresentaram dados da topografia pré-operatória no prontuário. Excluímos também olhos que implantaram outro tipo de LIO.

Analisando as 1.256 cirurgias pertencentes ao grupo LIO não tórica 61,30\% (770 olhos) foram excluídas por apresentarem astigmatismo menor que 1,00D, 24,6\% (309 olhos) por apresentarem dados insuficientes no prontuário (entre eles, falta de dados topográficos, refracionais e de acuidade visual) e 8,99\% (113 olhos) devido comorbidades que prejudicassem a avaliação da acuidade visual final.

Dentre os 196 olhos do grupo LIO tórica, 36,73\% (72 olhos) foram excluídos por dados insuficientes no prontuário e 19,89\% (39 olhos) devido patologias que interfiram na performance visual. Curioso salientar que um dos olhos foi excluído devido insatisfação do paciente após o implante de LIO tórica, e foi submetido à remoção cirúrgica da LIO, em tempo subsequente.

O projeto do estudo foi submetido à apreciação e aprovado pelo Comitê de Ética em Pesquisa da Sociedade Evangélica Beneficente de Curitiba, protocolado sob o número 4977/10, em 31 de maio de 2010.

No pré-operatório foram avaliados idade, sexo, dados topográficos do olho operado, e refração por método automatizado (Autorefrator Topcon RM8800, Japão). No período pós-operatório (um mês após a cirurgia), foram revisados dados da refração dinâmica e acuidades visuais com e sem correção. Realizou-se comparação do astigmatismo corneano pré-operatório (delta K pré) com o astigmatismo refracional pós-operatório (cilindro pós). Em pacientes com diagnóstico de rotação de LIO no pós-operatório (apenas 2 pacientes), os dados considerados foram aqueles após o reposicionamento da LIO.
Para calcular a média da acuidade visual e seu desvio padrão, utilizamos o equivalente em logMAR ${ }^{(13)}$.

Para análise da correlação entre as variáveis e idade utilizamos teste de Spearman. Para a comparação dos resultados em dois diferentes momentos em relação a variáveis quantitativas foi considerado o teste não-paramétrico de Wilcoxon. Para a comparação dos dois grupos definidos pelo tipo de lente (AcrySof ${ }^{\circledR}$ tórica e AcrySof ${ }^{\circledR} \mathrm{IQ}$ ), em relação a variáveis quantitativas, foi considerado o teste não-paramétrico de Mann-Whitney. A homogeneidade dos dois grupos em relação às distribuições de acuidade visual sem correção foi avaliada pelo teste de Qui-quadrado. Valores de $\mathrm{p}<0,05$ indicaram significância estatística. Os dados foram analisados com o programa computacional Statistica v.8.

\section{RESULTADOS}

A média da idade dos pacientes do grupo tórica foi de 65,6 anos e a do grupo não tórica foi de 72,1 anos. A distribuição do gênero foi semelhante em ambos os grupos. Não houve correlação entre idade e gênero $(p=0,309)$, nem entre idade e Delta K pré $(p=0,117)$.

Ao compararmos o astigmatismo corneano pré-operatório (Delta K pré) com o astigmatismo refracional pós-operatório residual (cilindro pós) observamos no grupo tórica uma redução estatisticamente significante $(p<0,001)$ (Tabela 1 e Gráfico 1). No grupo não tórica também houve uma tendência a redução, porém este número não foi significativo (Tabela 2 e Gráfico 1). Observamos que a redução média no grupo tórica foi igual a 1,37 dioptrias cilíndricas (DC) e no grupo não tórica foi igual a 0,16 DC.

Comparamos, também, a acuidade visual não corrigida (Tabela 3 e Gráfico 2) e corrigida (Tabela 4 e Gráfico 3) em ambos os grupos no período pós-operatório. Nas duas situações o grupo tórica apresentou melhor acuidade visual, com significância estatística. Acuidade visual expressa em logMAR.

\section{Tabela 1. Grupo tórica}

\begin{tabular}{lccccccc}
\hline & n & Média & Mediana & Mínimo & Máximo & $\begin{array}{c}\text { Desvio } \\
\text { padrão }\end{array}$ & $\begin{array}{c}\text { Valor } \\
\text { de p* }\end{array}$ \\
\hline Delta K (pré) & 85 & 2,10 & 1,79 & 1,0 & 5,60 & 1,05 & \\
Cilindro (pós) & 85 & 0,73 & 0,75 & 0,0 & 4,00 & 0,68 & $<0,001$ \\
Redução & 85 & 1,37 & 1,26 & $-1,8$ & 5,32 & 1,07 & \\
\hline
\end{tabular}

${ }^{*}=$ teste não-paramétrico de Wilcoxon, $p<0,05 ;{ }^{* *}=$ números expressos em dioptrias cilíndricas (DC).

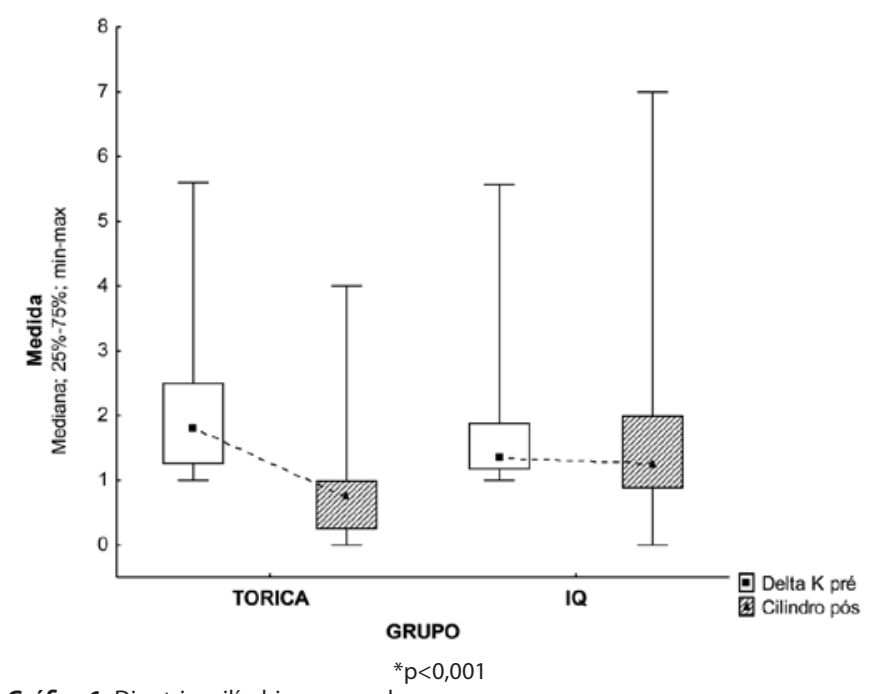

Gráfico 1. Dioptrias cilíndricas em ambos os grupos. 
Tabela 2. Grupo não tórica

\begin{tabular}{lccccccc} 
& n & Média & Mediana & Mínimo & Máximo & $\begin{array}{c}\text { Desvio } \\
\text { padrão }\end{array}$ & $\begin{array}{c}\text { Valor } \\
\text { de p* }\end{array}$ \\
\hline Delta K (pré) & 64 & 1,65 & 1,34 & 1,00 & 5,57 & 0,85 & \\
Cilindro(pós) & 64 & 1,49 & 1,25 & 0,00 & 7,00 & 1,08 & 0,057 \\
Redução & 64 & 0,16 & 0,22 & $-3,31$ & 2,50 & 0,93 & \\
\hline
\end{tabular}

${ }^{*}=$ teste não-paramétrico de Wilcoxon, $p<0,05 ;{ }^{* *}=$ números expressos em dioptrias cilíndricas (DC)

Tabela 3. Acuidade visual sem correção

\begin{tabular}{lccccccc}
\hline Grupo & N & Média & Mediana & Mínimo & Máximo & $\begin{array}{c}\text { Desvio } \\
\text { padrão }\end{array}$ & $\begin{array}{c}\text { Valor } \\
\text { de } \mathbf{p}^{*}\end{array}$ \\
\hline Tórica & 85 & 0,18 & 0,15 & 0,00 & 1,30 & 0,20 & \\
Não tórica & 64 & 0,49 & 0,50 & 0,00 & 2,00 & 0,33 & $<0,001$ \\
\hline
\end{tabular}

* = teste não-paramétrico de Mann-Whitney, $\mathrm{p}<0,05 ;{ }^{* *}=$ números expressos em logMAR.

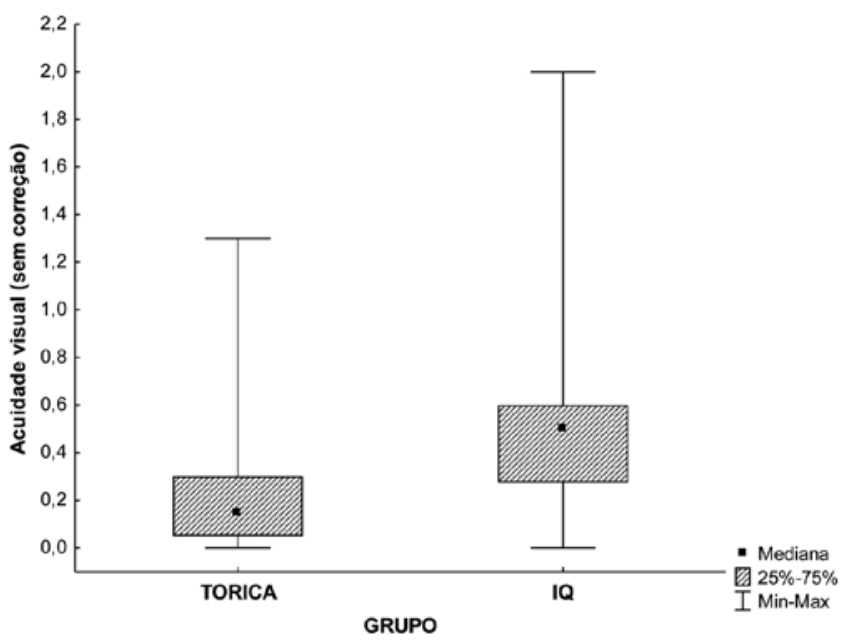

Gráfico 2. Acuidade visual sem correção.

\section{DISCUSSÃO}

Sabemos através dos resultados apontados por diversas pesquisas que a prevalência de astigmatismo na população geral gira em torno de 35 a $50 \%$ na maioria dos estudos ${ }^{(3,14-16)}$. Isto resulta em uma grande quantidade de pessoas que permanecem dependentes de óculos após a cirurgia de catarata quando ela corrige somente o componente esférico. Por isso, cada vez mais surgem novas tecnologias para a correção total das ametropias $s^{(5,7,8,10-12,17)}$, melhorando assim a qualidade de vida dos pacientes ${ }^{(7,8)}$.

$\mathrm{Na}$ atualidade, a cirurgia de facoemulsificação para a extração da catarata também é considerada para o paciente e para o cirurgião uma cirurgia refracional ${ }^{(6,7,7,10-12)}$. Por este motivo decidimos comparar os resultados cirúrgicos dos pacientes com astigmatismo maior ou igual a $1 \mathrm{D}$ que implantaram lente AcrySof ${ }^{\circledR}$ tórica e os que implantaram lente AcrySof ${ }^{\circledR} \mathrm{IQ}$.

Erros refrativos residuais após a cirurgia de catarata geralmente decorrem do cálculo biométrico imperfeito, inadequação da fórmula usada, posicionamento inadequado da lente e da presença de astigmatismo pré-operatório(1).

Observamos neste estudo que os pacientes do grupo tórica obtiveram uma redução do astigmatismo médio (Delta K pré versus cilindro pós) de 1,37 D em comparação a 0,16 D no grupo não tórica
Tabela 4. Acuidade visual com correção

\begin{tabular}{lccccccc}
\hline Grupo & N & Média & Mediana & Mínimo & Máximo & $\begin{array}{c}\text { Desvio } \\
\text { padrão }\end{array}$ & $\begin{array}{c}\text { Valor } \\
\text { de } \mathbf{p}^{*}\end{array}$ \\
\hline Tórica & 85 & 0,05 & 0,00 & 0,00 & 0,50 & 0,09 & \\
Não tórica & 64 & 0,12 & 0,10 & 0,00 & 0,50 & 0,12 & 0,003 \\
\hline
\end{tabular}

* = teste não-paramétrico de Mann-Whitney, $\mathrm{p}<0,05$; $^{* *}=$ números expressos em logMAR.

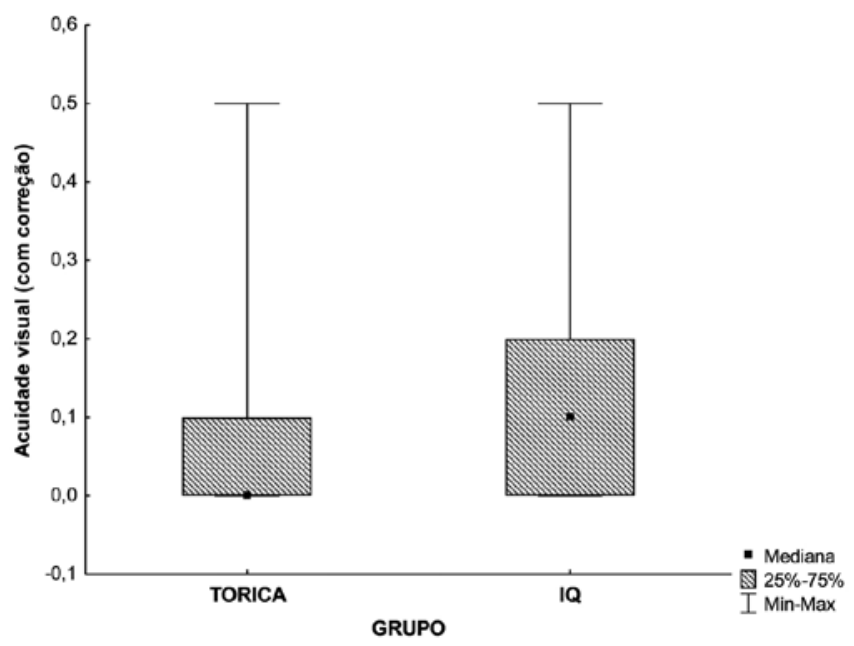

Gráfico 3. Acuidade visual com correção.

$(p<0,001)$. Esta diferença leva a uma melhora importante na performance visual final dos pacientes com este tipo de implante. Na prática, podemos observar a significância destes valores quando comparamos a acuidade visual sem correção (AVSC) destes dois grupos.

Sabemos que no paciente pseudofácico a origem principal do astigmatismo refracional é a córnea. O Delta K pré foi maior no grupo tórica (2,10 DC) do que no grupo não tórica (1,65 DC). Ainda assim, o astigmatismo refracional pós-operatório foi menor nos pacientes do grupo tórica. Demonstra-se, desta forma, que esta redução não ocorreu simplesmente ao acaso. O implante de LIO tórica foi determinante para que se obtivesse uma diminuição do astigmatismo refracional no período pós-operatório.

Vale lembrar que as incisões confeccionadas para a realização da facoemulsificação e implante da LIO alteram pouco a curvatura corneana ${ }^{(17,18)}$. Assumimos, teoricamente, que a cirurgia alterou a curvatura corneana de ambos os grupos de forma similar.

Ao considerarmos a AVSC, o grupo tórica apresentou em 44 olhos $(51,7 \%)$ AVSC de 0 logMAR (20/20) ou 0,1 logMAR (20/25), enquanto apenas 7 olhos (10,93\%) do grupo não tórica apresentaram este mesmo resultado.

Sanders et al. também já demonstraram que o implante de LIO tórica é eficaz e previsível, inclusive nos casos de moderados e altos astigmatismos $^{(7)}$. O estudo Europeu Multicêntrico corrobora com nossos resultados, pois apresentou ganho de linhas na tabela de Snellen com implantes intraoculares tóricos, já em 2003 ${ }^{(9)}$. Análises vetoriais recentes também demonstraram uma correção efetiva do astigmatismo com LIO's tóricas ${ }^{(19)}$.

Devemos lembrar que a rotação da LIO é uma das intercorrências que teremos de lidar com alguns pacientes. A técnica cirúrgica para implante de LIO's tóricas exige planejamento pré e intraoperatórios adequados, visto que estas LIO's devem ser colocadas no eixo correto para correção do astigmatismo, e permanecer assim no período pós-cirúrgico. Uma biometria de precisão é fundamental neste cenário. Mesmo com todos estes cuidados, a rotação das LIO's pode 
ocorrer. Nestes casos, o reposicionamento precoce traz melhores resultados ${ }^{(20,21)}$. Roturas zonulares ou quaisquer outras alterações que comprometam a centralização precisa da LIO no saco capsular podem contraindicar seu implante.

Com a evolução das técnicas cirúrgicas e variedade de lentes intraoculares disponíveis, a independência do uso de óculos passou a se tornar uma expectativa dos pacientes. Com as técnicas cirúrgicas atuais ainda não podemos assegurar tal independência. Porém, cada avanço tecnológico neste sentido é uma conquista.

Em conclusão, pudemos observar diminuição estatisticamente significativa do astigmatismo refracional pós-cirúrgico nos pacientes submetidos a facoemulsificação com implante de LIO tórica. Mostrou-se, também, uma técnica efetiva, previsível e relativamente simples. Esperamos que com o advento das lentes multifocais tóricas possamos ofertar uma excelente performance visual, tanto para longe, quanto para perto de forma satisfatória.

\section{REFERÊNCIAS}

1. Ventura LO, Barros EA, Arruda Junior JR. Sinais e sintomas das ametropias. In: Schor P, Uras R, Veitzman S. Óptica, refração e visão subnormal. Rio de Janeiro: Cultura Médica; 2008. p.205-17. Série Oftalmologia Brasileira.

2. Alves MR, Polati M, Faria e Sousa SJ. Refratometria ocular e a arte da prescrição médica. Rio de Janeiro: Cultura Médica; 2009.

3. Rayes TR, Rayes GR, Eing F, Gumarães Neto HP, Marquardt FA, Rayes A. Prevalência do astigmatismo e distribuição de seu eixo em pacientes de um serviço oftalmológico privado. Rev Bras Oftalmol. 2007;66(6):396-75.

4. Gills JP. Treating astigmatism at the time of cataract surgery. Curr Opin Ophthalmol. 2002;13(1):2-6

5. Rocha RC, Oechsler RA, Carvalho RG, Moreira H. Influência do astigmatismo corneano na acuidade visual final após implante de AcrySof ${ }^{\oplus}$ ReSTOR ${ }^{\oplus}$ : relato de caso. Arq Bras Oftalmol. 2007;70(6):1040-2.

6. Silva EF, Trindade FC. Correção do astigmatismo na cirurgia da catarata. Arq Bras Oftalmol. 2007;70(4):609-14.

7. Sanders DR, Schneider D, Martin R, Brown D, Dulaney D, Vukich J, et al. Toric Im- plantable Collamer Lens for moderate to high myopic astigmatism. Ophthalmology. 2007;114(1):54-61.

8. Correia RJ, Moreira H, Netto SU, Pantaleão GR. Visual performance after toric IOL implantation in patients with corneal astigmatism. Arq Bras Oftalmol. 2009;72(5): 636-40.

9. Dick HB, Alió J, Bianchetti M, Budo C, Christiaans BJ, El-Danasoury MA, et al. Toric phakic intraocular lens: European multicenter study. Ophthalmology. 2003;110(1):150-62.

10. Nichamin LD. Astigmatism management for modern phaco surgery. Int Ophthalmol Clin. 2003;43(3):43-63.

11. Nichamin LD. Treating astigmatism at the time of cataract surgery. Curr Opin Ophthalmol. 2003;14(1)35-8. Review.

12. Hida WT, Motta $A F$, Inomata $D L$, Jales $M Q$, Facio Júnior $A C$, José Júnior $N K$, et al. Incisões relaxantes limbares ou incisões no meridiano mais curvo associadas a facoemulsificação com implante de lente intra-ocular multifocal: relato de três casos. Arq Bras Oftalmol. 2008;71(2):273-7.

13. Holladay JT. Proper method for calculating average visual acuity. J Refract Surg. 1997; 13(4):388-91.

14. Attebo K, Ivers RQ, Mitchell P. Refractive errors in an older population: the Blue Mountains Eye Study. Ophthalmology. 1999;106(6):1066-72.

15. Bourne RR, Dineen BP, Ali SM, Noorul Huq DM, Johnson GJ. Prevalence of refractive error in Bangladeshi adults: results of the National Blindness and Low Vision Survey of Bangladesh. Ophthalmology. 2004;111(6):1150-60.

16. Fledelius HC. Prevalences of astigmatism and anisometropia in adult danes. With reference to presbyopes' possible use of supermarket standard glasses. Acta Ophthalmol (Copenh). 1984;62(3):391-400.

17. Tejedor J, Pérez-Rodríguez JA. Astigmatic change induced by $2.8-\mathrm{mm}$ corneal incisions for cataract surgery. Invest Ophthalmol Vis Sci. 2009;50(3):989-94.

18. Giansanti F, Rapizzi E, Virgili G, Mencucci R, Bini A, Vannozzi L, et al. Clear corneal incision of $2.75 \mathrm{~mm}$ for cataract surgery induces little change of astigmatism in eyes with low preoperative corneal cylinder. Eur J Ophthalmol. 2006;16(3):385-93.

19. Visser N, Berendschot TT, Bauer NJ, Nuijts RM. Vector analysis of corneal and refractive astigmatism changes following toric pseudophakic and toric phakic IOL implantation. Invest Ophthalmol Vis Sci. 2012;53(4):1865-73.

20. Amesbury EC, Miller KM. Correction of astigmatism at the time of cataract surgery. Curr Opin Ophthalmol. 2009;20(1):19-24. Review.

21. Kersey JP, O'Donnell A, Illingworth CD. Cataract surgery with toric intraocular lenses can optimize uncorrected postoperative visual acuity in patients with marked corneal astigmatism. Cornea. 2007;26(2):133-5. 